\title{
REPRODUTIBILIDADE INTRA E INTEROBSERVADORES DA CLASSIFICACÃO DE HIPERSINAL FACETÁRIO LOMBAR E CORRELAÇÃO COM A DEGENERAÇÃO DISCAL PARA RESSONÂNCIA MAGNÉTICA
}

\author{
INTRAOBSERVER AND INTEROBSERVER REPRODUCIBILITY OF LUMBAR FACET \\ HYPERSIGNAL RATING AND CORRELATION WITH DISC DEGENERATION BY \\ MAGNETIC RESONANCE IMAGING \\ REPRODUCIBILIDAD INTRA E INTER OBSERVADORES DE LA CLASIFICACIÓN DE
HIPERSENAL FACETARIA LUMBAR Y CORRELACIÓN CON LA DEGENERACIÓN
DISCAL PARA RESONANCIA MAGNÉTICA
}

André Evaristo Marcondes César ${ }^{1}$, Adriano Masayuki Yonezakl ${ }^{2}$, Fabrício Hidetoshi Ueno², Edgar Santiago Valesin Filho², LuCiano Miller Reis Rodrigues ${ }^{3}$

\begin{abstract}
RESUMO
Objetivo: Avaliar a reprodutibilidade intra e interobservadores da "classificação de hipersinal facetário lombar" e avaliar a correlação com a degeneração discal. Métodos: Estudo retrospectivo de imagens de ressonância magnética obtidas de 41 ( $N=41)$ pacientes (18 homens e 23 mulheres), com idade variando de 26 a 84 anos, com média de 48 anos e três meses. As imagens foram revisadas por três ortopedistas especialistas em cirurgia de coluna e um radiologista, para avaliar e quantificar a presença do hipersinal facetário lombar. Posteriormente, foi verificada a relação entre a artrose facetária e degeneração discal. Resultados: Foram avaliados e classificados 205 discos lombares e suas facetas articulares pelos quatro examinadores, com os dados analisados pelo Teste de Cronbach e da Análise de Correlação de Spearman com resultados estatisticamente elevados, confirmando boa relação intra e interobservadores para a "classificação de hipersinal facetário". Não foi observada relação estatisticamente significante entre artrose facetária e degeneração discal. Conclusão: Foram obtidos resultados suficientes para afirmar que existe boa relação intra e interobservadores para a "classificação de hipersinal facetário lombar". Não se verificou relação estatisticamente significante entre artrose facetária e degeneração discal.
\end{abstract}

Descritores: Sinovite; Coluna vertebral; Artrose; Imagem por ressonância magnética; Estudos retrospectivos.

\begin{abstract}
Objective: To assess intraobserver and interobserver reproducibility of lumbar facet hypersignal rating and to assess the correlation with disc degeneration. Methods: Retrospective study of magnetic resonance imaging of 41 patients (18 male and 23 female), with mean age of 48 years and three months (range 26-84 years). Three independent orthopaedic spine surgeons and one radiologist reviewed the images to assess and quantify the presence of lumbar facet hypersignal. Later, the relation between facet arthrosis and disc degeneration was evaluated. Results: 205 lumbar discs and facets were assessed and rated by four independent observers, the records were analyzed by Cronbach test and Spearman correlation analysis with high statistical outcomes which confirm a good interobserver and intraobserver relation for lumbar facet hypersignal rating. No statistical difference between facet arthrosis and disc degeneration was verified. Conclusion: Sufficient outcomes were obtained to confirm the existence of good interobserver and intraobserver relation for lumbar facet hypersignal rating. No statistical difference between facet arthrosis and disc degeneration was verified.
\end{abstract}

Keywords: Synovitis; Spine; Arthrosis; Magnetic resonance imaging; Retrospective studies.

\section{RESUMEN}

Objetivo: Evaluar la reproducibilidad inter e intra obsenvadores de la "Clasificación de hiperseñal facetaria lumbar" y evaluar la correlación con la degeneración discal. Métodos: Estudio retrospectivo de imágenes de resonancia magnética obtenidas de 41 ( $N=41)$ pacientes (18 hombres y 23 mujeres), con la edad de 26 años hasta 84 años y, con promedio de 48 años y 3 meses. Las imágenes fueran revisadas por tres cirujanos ortopedistas especialistas en cirugía de columna vertebral y un radiólogo para evaluar y cuantificar la presencia de hiperseñal facetario lumbar. Después fue verificada la relación entre la artrosis facetaria y la degeneración del disco. Resultados: Fueran evaluados y clasificados 205 discos lumbares y sus facetas articulares por los cuatro examinadores con las informaciones analizadas por la Prueba de Cronbach y el Análisis de Correlación de Spearman, con resultados estadísticamente elevados, confirmando buena relación inter e intra observadores para la "clasificación de hiperseñal facetaria" . No fue observada relación estadísticamente significativa entre artrosis facetaria y degeneración discal. Conclusión: Fueran obtenidos resultados suficientes para asegurar que hay buena relación inter y intra observadores para la "Clasificación de hiperseñal facetaria". No fue verificada relación estadísticamente significativa entre artrosis facetaria y degeneración discal.

Descriptores: Sinovitis; Columna vertebral; Artrosis; Imagen por resonancia magnética; Estudios retrospectivos.

1. Médico Estagiário do Grupo de Cirurgia da Coluna Vertebral da Faculdade de Medicina do ABC - Santo André (SP), Brasil.

2. Médico Assistente do Grupo de Cirurgia da Coluna Vertebral da Faculdade de Medicina do ABC - Santo André (SP), Brasil.

3. Chefe do Grupo de Cirurgia de Coluna Vertebral da Faculdade de Medicina do ABC - Santo André (SP), Brasil.

Trabalho realizado no Departamento do Aparelho Locomotor da Faculdade de Medicina do ABC - Santo André, SP, Brasil.

Correspondência: Rua Borges Lagoa 1065, CEP 01455- 010 - E-mail luciano.miller@uol.com.br 


\section{INTRODUÇÃO}

A dor lombar é a segunda causa mais frequente de queixa em Pronto-Socorro, atrás apenas de doenças do aparelho respiratório $^{1,2}$. Cerca de $70 \%$ dos adultos tiveram pelo menos um episódio de dor lombar durante a vida, e 14\% nas duas últimas semanas $^{3}$. A dor lombar tem como origem mais frequente o disco intervertebral ${ }^{4}$.

As facetas articulares têm sido apontadas como outra importante causa de dor lombar ${ }^{5,6}$. A ressonância magnética proporciona uma maior precisão na determinação das alterações degenerativas dessas articulações ${ }^{7-9}$. Comumente esse processo degenerativo apresenta um hipersinal nas facetas articulares lombares, observadas nos cortes axial e sagital em imagens ponderadas em T27 . O aparecimento desse sinal é homogêneo em densidade mas, no entanto, varia em comprimento, porém contido nas margens articulares. Este é retilíneo, sem contornos irregulares, comumente associado a uma alteração degenerativa das articulações lombares. As margens da cápsula articular não se encontram excessivamente distendidas e não há evidência de acúmulo de líquido extra articular ${ }^{7}$

Yang e Yang ${ }^{10}$, em 2005, foram os primeiros autores a discutirem a presença desse hipersinal facetário, relatando a associação com a dor lombar e degeneração do disco intervertebral. Longmuir e Conley ${ }^{7}$ propuseram uma classificação para a presença do hipersinal facetário e definiram esse sinal como sendo um processo reacional das facetas articulares.

As alterações facetárias não têm sido estudadas como a degeneração discal, apesar de serem uma importante fonte de dor lombar. Em relação ao disco intervertebral, Pfirrmann et al. ${ }^{11}$ propuseram uma classificação que tem sido adotada para padronização de alterações discais, com boa reprodutibilidade demonstrada por Ueno et al. ${ }^{4}$

Nosso artigo tem como objetivo avaliar a reprodutibilidade da Classificação de hipersinal facetário e estudar a existência de correlação com a degeneração discal.

\section{MATERIAL E MÉTODO}

Foi analisado o segmento lombar compreendido entre L1 até S1 de 41 pacientes. $\mathrm{O}$ grupo foi formado por 18 homens e 23 mulheres, com idade variando de 26 a 84 anos com média de 48 anos e três meses.

A ressonância magnética utilizada foi de 1-tesla com corte axial ponderada em T2. Os pacientes inclusos no estudo estavam em tratamento ambulatorial devido à queixa clínica de lombociatalgia ou estenose do canal lombar (Figuras 1 e 2). Utilizamos a classificação de Longmuir and Conley para graduarmos a alteração das articulações facetarias ${ }^{7}$ (Quadro 1).

A avaliação da reprodutibilidade da classificação de hipersinal facetário foi realizada por quatro observadores: um ortopedista em treinamento para cirurgia de coluna, dois ortopedistas especialistas em cirurgia da coluna e um radiologista. Os observadores reavaliaram as imagens com um intervalo mínimo de uma semana.

A

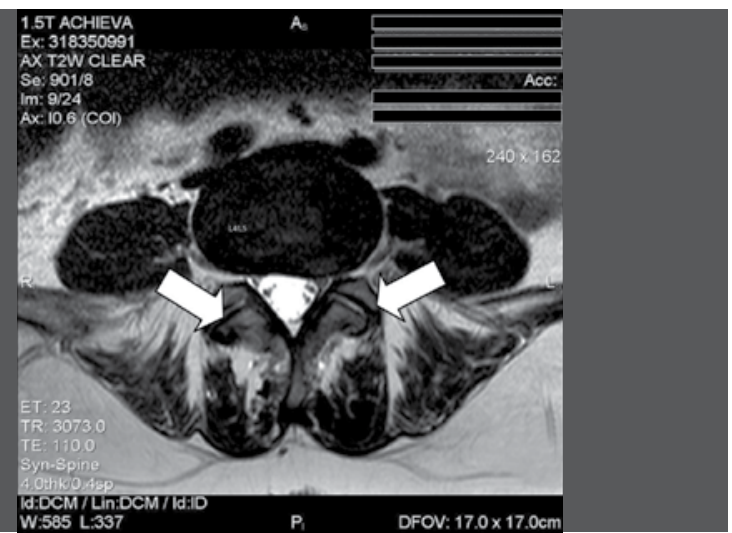



Figura 1. Brilho facetário presente em mulher de 29 anos de idade. (A): imagem axial. Note o envolvimento das facetas articulares direita e esquerda das articulações L4-L5. (B): corte sagital dos aspecto lateral à esquerda das facetas articulares de L4-L5 e L5-S1 com a presença de hipersinal.

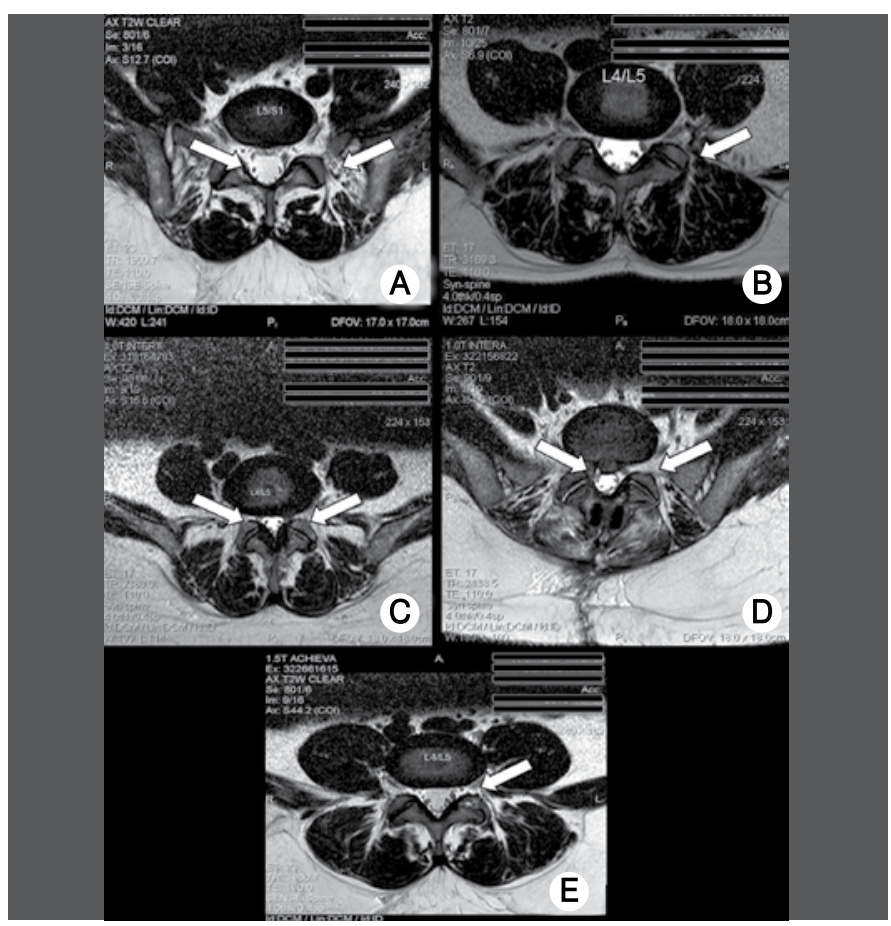

Figura 2. Classificação de hipersinal facetário. (A): grau 0 (faceta normal) (B): grau I (brilho facetário reponde por menos de $50 \%$ da faceta articular) (C): grau II (brilho facetário responde por mais de 50\% da faceta articular). (D): grau III (brilho facetário corresponde a $100 \%$ da faceta articular). (E): grau IV (erosão óssea).

A determinação do grau de degeneração discal foi obtida por consenso entre os observadores em conjunto, utilizando a classificação de Pfirrmann et al. ${ }^{11}$ para a graduação (Quadro 2). Após isso, testamos a sua relação com a classificação de hipersinal facetário.

$\mathrm{Na}$ análise estatistica foi utilizado o programa Statistical Package for Social Science (SPSS) em sua versão 17.0 para obtenção dos resultados com nível de significância $\mathrm{p}<0,05$. 
Quadro 1. Diferentes graus da "classificação de hipersinal facetário", variando da faceta normal até a erosão óssea.

\begin{tabular}{|c|l|}
\hline Grau & \multicolumn{1}{|c|}{ Definição } \\
\hline 0 & Faceta normal \\
\hline I & $\begin{array}{l}\text { Brilho facetário responde a menos de } 50 \% \text { do comprimento da } \\
\text { cartilagem hialina no corte axial }\end{array}$ \\
\hline II & $\begin{array}{l}\text { Brilho facetário responde a mais de } 50 \% \text { do comprimento da cartilagem } \\
\text { hialina no corte axial }\end{array}$ \\
\hline III & Brilho facetário responde a todo o comprimento da cartilagem hialina \\
\hline IV & Erosão óssea \\
\hline
\end{tabular}

Quadro 2. Classificação de degeneração discal de Pfirrmann ${ }^{11}$.

\begin{tabular}{|c|c|c|c|c|}
\hline Grau & Estrutura & $\begin{array}{c}\text { Distinção } \\
\text { de núcleo e } \\
\text { ânulo }\end{array}$ & Intensidade do sinal & Altura do disco \\
\hline I & $\begin{array}{c}\text { Homogênea, brando } \\
\text { brilhante }\end{array}$ & Nítido & $\begin{array}{c}\text { Hiperintenso, isointenso } \\
\text { para fluído liquórico }\end{array}$ & Normal \\
\hline II & $\begin{array}{c}\text { Não homogênea com ou } \\
\text { sem bandas horizontais }\end{array}$ & Nítido & $\begin{array}{c}\text { Hiperintenso, isointenso } \\
\text { para fluido liquórico }\end{array}$ & Normal \\
\hline III & Não homogênea, cinza & Não nítido & Intermediário & $\begin{array}{c}\text { Normal para } \\
\text { pouco diminuído }\end{array}$ \\
\hline IV & $\begin{array}{c}\text { Não homogênea, cinza } \\
\text { para preto }\end{array}$ & Perda & $\begin{array}{c}\text { Intermediário a } \\
\text { isointenso }\end{array}$ & $\begin{array}{c}\text { Normal para } \\
\text { moderadamente } \\
\text { diminuído }\end{array}$ \\
\hline V & Não homogênea, preto & Perda & Hipointenso & $\begin{array}{c}\text { Espaço discal em } \\
\text { colapso }\end{array}$ \\
\hline
\end{tabular}

\section{RESULTADO}

Na análise inter-observadores foi utilizado o teste da Estatística Alfa de Cronbach, observamos uma boa concordância entre os examinadores (Tabela 1). Permite-se considerar que a amostra demonstra graus de confiabilidade entre "satisfatório" e "elevado" (entre 0,639 a 0,904).

Para a análise intra-observadores foi utilizada a Análise de Correlação de Spearman, com o intuito de verificarmos se as correlações calculadas apresentam comportamentos semelhantes no "teste" e no "reteste" (momentos 1 e 2). Esse estudo apresentou variação estatística significante entre os dois momentos de observação (Tabela 2).

Não houve correlação estatisticamente significante entre a graduação de Pfirmman, e a Classificação de hipersinal facetário. Nessa verificação utilizamos a Análise de Correlação Estatística de Spearman (Tabela 3).

Tabela 1. Resultados da Estatística Alfa de Cronbach para a análise interobservadores (observar que todas as variantes apresentam nível de significância $p<0,05$.

\begin{tabular}{c|c|c}
\hline Aspecto & Coeficiente Alfa de Cronbach & Significância (p) \\
\hline $11-\mid 2(1)$ & 0,639 & $<0,001$ \\
\hline $12-\mid 3(1)$ & 0,873 & $<0,001$ \\
\hline $13-\mid 4(1)$ & 0,903 & $<0,001$ \\
\hline $14-15(1)$ & 0,844 & $<0,001$ \\
\hline $\mid 5-\mathrm{s} 1(1)$ & 0,876 & $<0,001$ \\
\hline $11-12(2)$ & 0,672 & $<0,001$ \\
\hline $12-13(2)$ & 0,875 & $<0,001$ \\
\hline $13-14(2)$ & 0,904 & $<0,001$ \\
\hline $14-15(2)$ & 0,848 & $<0,001$ \\
\hline $\mid 5-51(2)$ & 0,867 & $<0,001$ \\
\hline
\end{tabular}

Tabela 2. Resultados da Análise de Correlação de Spearman para a análise intra-observadores (observar que todas as variantes apresentam nível de significância $p<0,05)$.

\begin{tabular}{c|c|c}
\hline Pares de Variáveis & Coeficiente de Correlação & Significância (p) \\
\hline$|1|-|2(1) \times| 1-\mid 2(2)$ & 0,960 & $<0,001$ \\
\hline$|2-| 3(1) \times|2-| 3(2)$ & $>0,999$ & $<0,001$ \\
\hline$|3-| 4(1) \times|3-| 4(2)$ & $>0,999$ & $<0,001$ \\
\hline$|4-15(1) \times| 4-15(2)$ & $>0,999$ & $<0,001$ \\
\hline$|5-s 1(1) \times| 5-\mathrm{s} 1(2)$ & 0,954 & $<0,001$ \\
\hline
\end{tabular}

Tabela 3. Resultados da Análise de Correlação de Spearman para avaliar a relação entre a classificação de hipersinal facetário lombar e a graduação de Pfirrmann ${ }^{11}$

\begin{tabular}{|c|c|c|c|c|c|c|}
\hline Variável & Estatística & $\begin{array}{c}11-12 \\
\text { Consenso } \\
\text { PFI } \\
\end{array}$ & $\begin{array}{c}\mid 2-13 \\
\text { Consenso } \\
\text { PFI } \\
\end{array}$ & $\begin{array}{c}13-14 \\
\text { Consenso } \\
\text { PFI } \\
\end{array}$ & $\begin{array}{c}4-15 \\
\text { Consenso } \\
\text { PFI } \\
\end{array}$ & $\begin{array}{c}\text { I5-s1 } \\
\text { Consenso } \\
\text { PFI } \\
\end{array}$ \\
\hline \multirow{3}{*}{$\begin{array}{l}\text { I1-I2 Andre } \\
\text { CHF } 1\end{array}$} & \begin{tabular}{|c|} 
Coef. Correl. \\
$(r)$
\end{tabular} & 0,060 & 0,111 & 0,053 & $-0,060$ & 0,030 \\
\hline & Sig. (p) & 0,714 & 0,496 & 0,744 & 0,713 & 0,853 \\
\hline & N & 40 & 40 & 40 & 40 & 40 \\
\hline \multirow{3}{*}{$\begin{array}{c}\text { I2-I3 Andre } \\
\text { CHF } 1\end{array}$} & \begin{tabular}{|c|}
$\begin{array}{c}\text { Coef. Correl. } \\
(r)\end{array}$ \\
\end{tabular} & 0,078 & 0,133 & 0,224 & 0,190 & $-0,007$ \\
\hline & Sig. (p) & 0,648 & 0,433 & 0,182 & 0,260 & 0,966 \\
\hline & N & 37 & 37 & 37 & 37 & 37 \\
\hline \multirow{3}{*}{$\begin{array}{c}\text { I3-14 Andre } \\
\text { CHF } 1\end{array}$} & \begin{tabular}{|c} 
Coef. Correl. \\
$(\mathrm{r})$
\end{tabular} & 0,088 & 0,036 & $-0,140$ & 0,032 & 0,072 \\
\hline & Sig. (p) & 0,594 & 0,827 & 0,395 & 0,847 & 0,664 \\
\hline & N & 39 & 39 & 39 & 39 & 39 \\
\hline \multirow{3}{*}{$\begin{array}{l}\text { 14-15 Andre } \\
\text { CHF } 1\end{array}$} & \begin{tabular}{|c|}
$\begin{array}{c}\text { Coef. Correl. } \\
\text { (r) }\end{array}$ \\
\end{tabular} & 0,191 & 0,240 & 0,453 & 0,101 & 0,129 \\
\hline & Sig. (p) & 0,252 & 0,146 & 0,004 & 0,544 & 0,439 \\
\hline & N & 38 & 38 & 38 & 38 & 38 \\
\hline \multirow{3}{*}{$\begin{array}{c}\text { 15-s1 Andre } \\
\text { CHF } 1\end{array}$} & \begin{tabular}{|c|}
$\begin{array}{c}\text { Coef. Correl. } \\
(r)\end{array}$ \\
\end{tabular} & 0,193 & 0,105 & 0,070 & $-0,136$ & $-0,007$ \\
\hline & Sig. (p) & 0,247 & 0,530 & 0,675 & 0,416 & 0,969 \\
\hline & N & 38 & 38 & 38 & 38 & 38 \\
\hline
\end{tabular}

\section{DISCUSSÃO}

A artrose facetária tem sido sugerida como causa de dor lombar $^{1,12}$. No entanto, a origem da dor facetária ainda não foi bem elucidada. Teorias incluem alterações mecânicas, vasculares e moleculares. Enquanto alguns pacientes durante o processo de degeneração articular evoluem com quadro de dor lombar baixa, outros somente evoluem com dor quando ocorre o desalinhamento $\operatorname{lombar}^{13}$. As principais alterações patológicas atribuídas à faceta articular degenerada são estreitamento do espaço articular, esclerose do osso subcondral, formação de osteófitos e hipertrofia articular ${ }^{14}$.

Na coluna lombar existe uma significante variação no volume do fluído, que é o responsável pelo "brilho" facetário. As sinovites são caracterizadas por hiperemia e infiltrado inflamatório no tecido sinovial, podendo contribuir para o aparecimento de "hipersinal facetário"7.

No estudo de articulação temporomandibular de Segami et al. ${ }^{15}$ foi relatado que articulações que apresentam efusão de fluídos possuem mais sinovite que articulações sem efusão. A presença de efusão articular nas imagens de ressonância magnética ponderadas em T2 geralmente refletem a presença de sinovite ${ }^{15}$.

Segundo o artigo de Manchikanti et al. ${ }^{16}$, há uma correlação entre a doença degenerativa discal e facetária colocando como hipótese que existe uma correlação entre a alteração anterior e 
posterior da unidade funcional da coluna. No presente estudo não foi observada relação entre o grau de artrose facetária e degeneração discal utilizando as diferentes classificações para obter essa graduação.

No artigo de Longmuir e Conley ${ }^{7}$ foi relatada boa concordância intra e interobservadores na graduação da classificação de hipersinal facetário. Nosso estudo também demonstrou a boa correlação da classificação de Longmuir e Conley entre os examinadores testados. A aplicabilidade dessa classificação será importante se futuros estudos demonstrarem uma correlação com alterações clínicas, para podermos ser mais específicos na indicação de tratamento.

$\mathrm{Na}$ degeneração do segmento funcional da coluna o grau de degeneração discal é bem definido e, mais recentemente, o grau de alteração facetária também tem sido classificado. Nosso artigo testou a correlação entre as duas classificações não demonstrando uma boa correlação. A tentativa de relacionar as classificações é extremamente importante principalmente com o advento da medicina regenerativa, para podermos analisar se terapias discais promovem alguma modificação nas facetas articulares e, também, como forma de padronização de modelo de artrose facetária para estudos experimentais.

\section{CONCLUSÃO}

A "Classificação de hipersinal facetário" constatou ser um sistema de boa aplicabilidade na diferenciação entre os graus de artrose facetária. No entanto, não foi encontrada relação estatisticamente significante com a degeneração discal.

\section{REFERÊNCIAS}

1. Eubanks JD, Lee MJ, Cassinelli E, Ahn NU. Prevalence of lumbar facet arthrosis and its relationship to age, sex, and race: an anatomic study of cadaveric specimens. Spine (Phila Pa 1976). 2007;32(19):2058-62.

2. Brodke DS, Ritter SM. Nonsurgical management of low back pain and lumbar disk degeneration. Instr Course Lect. 2005;54:279.

3. Deyo RA, Rainville J, Kent DL. What can the history and physical examination tell us about low back pain? JAMA. 1992:268(6):760-5

4. Ueno FH, Rodrigues LMR, Valesin Filho ES, Puertas EB. Reprodutibilidade intra e inter-observadores da classificação de Pifirrmann para degeneração discal por meio da ressonância magnética. Coluna/Columna. 2008;7(4):330-3

5. Goldthwait JE. The lumbosacral articulation: an explanation of many cases of lumbago, sciatica and paraplegia. Boston Med Surg J. 1911;164:365.

6. Ghormley RK. Low back pain with special reference to the articular facets with preservation of an operative procedure. JAMA. 1933;101:1773-7.

7. Longmuir GA, Conley RN. Interexaminer reliability of T2-weighted magnetic resonance imaging for lumbar bright facet sign. J Manipulative Physiol Ther. 2008;31 (8):593-601.

8. Modic MT, Masaryk TJ, Ross JS, Carter JR. Imaging of degenerative disk disease. Radiology. 1988;168(1):177-86.

9. Peterfy CG. MR imaging. Baillieres Clin Rheumatol. 1996;10(4):635-78.
10. Yang SC, Yang PH. Significance of the bright facet sign on T2W MRI of lumbar facet joint. Mid Taiwan J Med. 2005;10:150-4.

11. Pfirrmann CW, Metzdorf A, Zanetti M, Hodler J, Boos N. Magnetic resonance classification of lumbar intervertebral disc degeneration. Spine (Phila Pa 1976). $2001 ; 26(17): 1873-8$

12. Kalichman L, Li L, Kim DH, Guermazi A, Berkin V, O'Donnell CJ, et al. Facet joint osteoarthritis and low back pain in the community-based population. Spine (Phila Pa 1976). 2008;33(23):2560-5

13. Giles LG, Taylor JR. Osteoarthrosis in human cadaveric lumbo-sacral zygapophyseal joints. J Manipulative Physiol Ther. 1985;8(4):239-43.

14. Kettler A, Werner K, Wilke HJ. Morphological changes of cervical facet joints in elderly individuals. Eur Spine J. 2007;16(7):987-92.

15. Segami N, Nishimura M, Kaneyama K, Miyamaru M, Sato J, Murakami KI. Does joint effusion on T2 magnetic resonance images reflect synovitis? Comparison of arthroscopic findings in internal derangements of the temporomandibular joint. Oral Surg Oral Med Oral Pathol Oral Radiol Endod. 2001;92(3):341-5.

16. Manchikanti L, Singh V, Pampati V, Damron KS, Beyer CD, Barnhill RC. Is there correlation of facet joint pain in lumbar and cervical spine? An evaluation of prevalence in combined chronic low back and neck pain. Pain Physician. 2002;5(4):365-71. 